\title{
Açaí (Euterpe olecareae Martius) intake and its protective effect against 1,2-DMH-induced rat colon carcinogenesis
}

\author{
Mariana F Fragoso ${ }^{1}$, Luís F Barbisan ${ }^{2 *}$ \\ From São Paulo Advanced School of Comparative Oncology \\ Águas de São Pedro, Brazil. 30 September - 6 October 2012
}

\section{Background}

Açaí (Euterpe olecareae Martius) is an important crop consumed in Brazil that gained attention due to its potential antioxidant and anti-inflammatory properties. Various studies have shown that this exotic fruit may reduce the risk of many diseases, including cancer. The present study investigated the beneficial potential of açaí extract intake on the 1,2-dimethylhydrazine (DMH)induced rat colon carcinogenesis.

\section{Materials and methods}

Five groups were studied: G1 to G4 groups received four s.c. injections of DMH $(40 \mathrm{mg} / \mathrm{kg})$ twice a week during two weeks and G5 group received similar injections of EDTA (DMH vehicle). After two weeks of colon initiation, groups were fed basal diet (G1), basal diet containing 2.5\% açaí extract (G2), basal diet containing $5.0 \%$ açaí extract (G3 and G5) or basal diet containing $0.2 \%$ of N-acetylcysteine (G4, positive control). Ten weeks after the beginning of the treatment, G1 to G4 groups were sacrificed for aberrant crypt foci (ACF) assay. Also, G1 and G3 groups were maintained on basal diet or basal diet containing $5.0 \%$ açaí extract, respectively, until week 20. Colons were analyzed for ACF development (week 10) or tumor incidence, multiplicity and histological analyses (week 20).

\section{Results}

The number of total aberrant crypts (AC) was significantly reduced in G3 and G4 groups when compared to G1 group $(p=0.036)$. Data from ACF (1-3 AC) was significantly lower in G3 and G4 groups ( $p=0.011$ and $p=0.037$, respectively). In the colon tumor assay, G1 group developed invasive $(75 \%)$ and noninvasive (25\%) tumors whereas G3 group developed only noninvasive tumors (100\%).

\section{Conclusions}

These results suggest that açaí extract intake is potentially beneficial for early reduction of the number of $\mathrm{AC}$ and may affect tumor development and late malignancy in male Wistar rats initiated for colon carcinogenesis.

\section{Financial support}

\section{CAPES}

\section{Author details}

'Department of Pathology, Botucatu Medical School, UNESP, Botucatu, SP, Brazil. ${ }^{2}$ Department of Morphology, Botucatu Biosciences Institute, UNESP, Botucatu, SP, Brazil.

Published: 4 April 2013

doi:10.1186/1753-6561-7-S2-P48

Cite this article as: Fragoso and Barbisan: Açaí (Euterpe olecareae Martius) intake and its protective effect against 1,2-DMH-induced rat colon carcinogenesis. BMC Proceedings 2013 7(Suppl 2):P48.

\footnotetext{
* Correspondence: barbisan@ibb.unesp.br

${ }^{2}$ Department of Morphology, Botucatu Biosciences Institute, UNESP,

Botucatu, SP, Brazil

Full list of author information is available at the end of the article
} 